\title{
Decoherence and Programmable Quantum Computation
}

\author{
Jeff P. Barnes and Warren S. Warren \\ Chemistry Department, Princeton University, Princeton, NJ 08544-1009
}

(August 7, 2021)

\begin{abstract}
An examination of the concept of using classical degrees of freedom to drive the evolution of quantum computers is given. Specifically, when externally generated, coherent states of the electromagnetic field are used to drive transitions within the qubit system, a decoherence results due to the back reaction from the qubits onto the quantum field. We derive an expression for the decoherence rate for two cases, that of the single-qubit Walsh-Hadamard transform, and for an implementation of the controlled-NOT gate. We examine the impact of this decoherence mechanism on Grover's search algorithm, and on the proposals for use of error-correcting codes in quantum computation.
\end{abstract}

PACS number(s): 03.67.Lx, 32.80.Qk 


\section{Introduction}

In the original concept of quantum computation, the isolated, coherent evolution of a quantum system corresponded to a series of logical operations which could be used to compute a solution to a problem [1]. In this concept, once a method of solving a given problem is decided upon, the logical steps in the method are translated into unitary transforms of a quantum system [2]. These transforms demand a certain form for the Hamiltonian $H$ that describes the evolution of the quantum computer, which in turn constrains the architecture of the quantum system. In other

words, the program determines the system propagator, $U(t)=\exp (-i H t / \hbar)$, which determines the form of $H$, whose parameters indicate the qubit-qubit interactions that must be present in order to carry out the program.

Such quantum computers suffer from a significant drawback: they are not programmable. Consider the case of NMR quantum computing. A given program to solve a given problem determines $H$, which in turn determines the magnitudes of the $J$ couplings between distinct spins, which in turn constrains the geometry of the molecule to be used as the quantum system. Once the molecule is created, perhaps utilizing organic synthetic chemistry, it is in general useful only for the method of solving the problem originally decided upon. In contrast with the flexibility of classical, transistor-based computers, these quantum computers have their programs "hard-wired" into their architecture.

However, recent proposals for quantum computer architectures seek to overcome this limitation. 
They use external fields, generated by classical degrees of freedom, in order to drive the quantum system's evolution [3]. Since classical sources can easily be manipulated by the (presumably classical) programmer, these methods offer a means by which the programmer can alter the evolution of the quantum system, and thus program the computer. Some of these proposals include the use of radio-frequency pulses acting upon nuclear spins in liquids [4, 5], laser pulses acting upon ions trapped in resonators [6, 7], electrostatic fields generated from gated electrodes influencing the evolution of nuclear spins in semiconductors $[\mathbb{8}$, or electrons trapped in quantum dot structures [9].

There is a question that naturally arises when one contemplates such proposals: how can the evolution of a quantum system remain coherent if it is interacting with classical degrees of freedom? Consider the loss of visibility of the interference pattern in a two-slit electron beam experiment which occurs when one attempts to measure which path the electron took using a light source [10]. The interaction of the field with the electron creates an entangled state of the electron and field, from which a measurement of the state of the light will reveal information about the position of the electron. To the extent to which the light carries information about which path the electron takes, interference is lost 10, 11, 12]. Such interference is central to the working of many quantum programs, e.g. Grover's search algorithm [13]. If we use classically generated fields to drive (and thus interact) with the qubits, will that interaction effectively measure the state of the qubits, and by doing so, destroy the coherent evolution of the system?

To answer this question, we examine a specific case, that of Grover's quantum search algorithm 
[13]. First, we review the implementation of this algorithm when quantum back reaction is not a concern. Then we assume that one of the transforms used in the algorithm, the Walsh-Hadamard transform, is driven by externally applied electromagnetic pulses. The quantum field is assumed to be generated by classical sources, so that it is described by a coherent field state. Thus, the quantum field exhibits behavior that is close to a classical field. Despite this, there is some quantum backreaction on the field which leads to a decoherence of the qubit system. We determine the rate of decoherence due to this process. Finally, we discuss the broader implications of this mechanism of decoherence. While these questions have been previously raised [14], to the author's knowledge no quantitative assessment of their importance has yet been given.

\section{Grover's algorithm}

\subsection{Discussion}

Before we consider how to drive a part of Grover's search algorithm, let us briefly review it here. Grover's quantum search algorithm is a method by which to retrieve elements in a subset of a larger set [13]. It acts upon $K$ qubits, or two-level systems, whose levels are arbitrarily label as $|0\rangle$ and $|1\rangle$. A complete, orthonormal basis for the system is the product basis, e.g. for $K=3,\{|000\rangle,|001\rangle$, $|010\rangle,|011\rangle,|100\rangle,|101\rangle,|110\rangle,|111\rangle\}$. It is usual to refer to each element of the basis set by the integer whose binary expansion represents the string of 0 s and 1 s, so that $|3\rangle \equiv|011\rangle$. The elements 
of the set to be searched are labeled by the integers from 0 to $2^{K}-1$.

The subset whose elements we are searching for is specified according to a condition. For example, we could search for any integer-valued roots of a given polynomials within a fixed range. There are many problems for which, given an $x$ of the set of possible solutions, it can be checked in a polynomial number of steps whether $x$ is a solution to the problem, but no known method exists to find all solutions in polynomial steps [15]. These problems can be solved by a brute-force search over all possible solutions, which is what Grover's algorithm does. Since Grover's algorithm has been shown to be optimal [16, 17, 18], its performance is one important indicator of how quantum computation might out-perform classical computation.

In what follows, we assume there is only a single solution, $y$, to the problem. It is not difficult to generalize this to multiple solutions [17]. The initial state of the quantum computer is $\sum_{x=0}^{2^{K}-1}|x\rangle / \sqrt{2^{K}}$. The goal is to transfer amplitude into the state $|y\rangle$ [13] so that a measurement of the system yields the solution. This is achieved by a series of transforms of the form $(\mathcal{W R} \mathcal{W O})^{N}$. The unitary, Hermitian operator $\mathcal{W}=\prod_{n} \mathcal{W}(n)=\prod_{n} \sqrt{2}\left(\mathcal{S}_{x}(n)-\mathcal{S}_{z}(n)\right)$ is the Welsh-Hadamard transform. It consists of a product of transforms, acting independently on each qubit in the system. (When referring to a single qubit, we employ the Pauli spin operator notation as commonly used in magnetic resonance [19]. In particular, $\mathcal{S}_{\alpha}=|1\rangle\langle 1|$ and $\mathcal{S}_{\beta}=|0\rangle\langle 0|$ are projection operators, and $\mathcal{S}_{+}=|1\rangle\langle 0|$ and $\mathcal{S}_{-}=|0\rangle\langle 1|$ are raising and lowering operators for a single qubit.)

The operators $\mathcal{R}=-\mathbf{1}+2|0\rangle\langle 0|$ and $\mathcal{O}=\mathbf{1}-2|y\rangle\langle y|$ are diagonal in the product basis. The 
operator $\mathcal{O}$ is called the oracle. It is the only means by which the algorithm has knowledge of the solution. It would be the routine the flips the amplitude of the state $|x\rangle$, for example, if $x$ were the root of a given polynomial. The combination $\mathcal{W R W}$ can also be written as $-\mathbf{1}+$ $2\left(\sum_{x=0}^{2^{K}-1}|x\rangle\right)\left(\sum_{x=0}^{2^{K}-1}\langle x|\right) / 2^{K}$, which is called the invert-about-average step.

The algorithm can be understood as a combination of two inversions, the first about $|y\rangle$ and the second about the state with an equal amplitude for all basis states. The two inversions result in a rotation, transferring amplitude into $|y\rangle[13$, 18]. For any normalized state of the computer, $\sum_{x=0}^{2^{K}-1} a_{x}|x\rangle$, the application of the transformation $\mathcal{W R} \mathcal{W O}$ alters the state as follows:

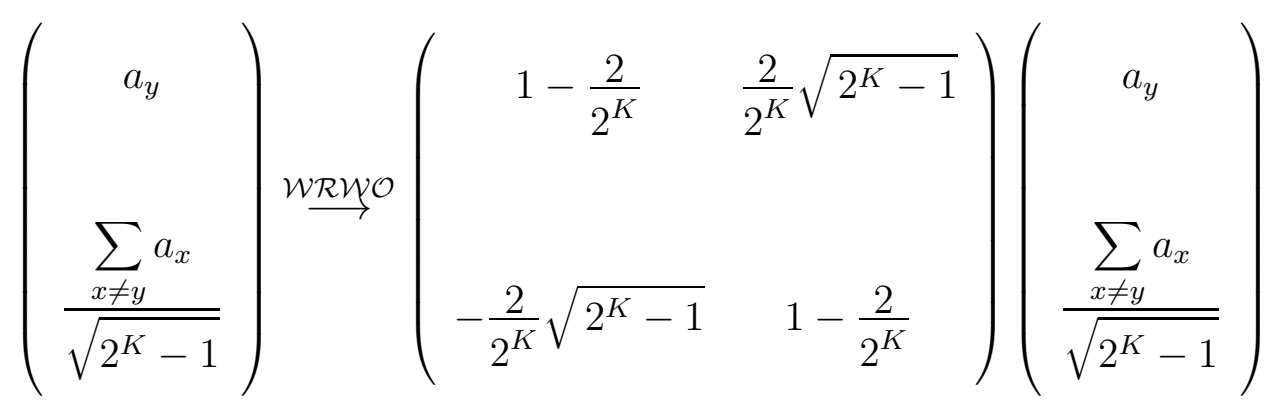

which is a rotation of the probability amplitude between $|y\rangle$ and all other states, with $\sin \varphi \approx 2^{1-K / 2}$ for large $K$. When $a_{y}$ and $\sum_{x \neq y} a_{x}$ have the same sign, the amplitude for the state $|y\rangle$ increases with every iteration, and decreases otherwise.

Note that $\mathcal{R}$ and $\mathcal{O}$, unlike $\mathcal{W}$, are not products of operators acting independently upon each qubit. They require qubit-qubit interactions to implement. To see this, write $\mathcal{R}=-\mathbf{1}+2 \prod_{n} \mathcal{S}_{\beta}(n)$ $=-\mathbf{1}+2 \prod_{n}\left[1 / 2-\mathcal{S}_{z}(n)\right]$. When the product is expanded, terms such as $\mathcal{S}_{z}(n) \mathcal{S}_{z}(m)$ appear, which indicate the need for qubit interactions. 


\subsection{Adding Classical Fields}

Consider how to drive the algorithm utilizing externally applied fields. The Walsh-Hadamard transforms $\mathcal{W}(n)$ can be driven one qubit at a time. Some proposals include methods by which qubit-qubit couplings, and thus $\mathcal{R}$ and $\mathcal{O}$, could also be driven [8, [1. We assume here that only the $\mathcal{W}(n)$ are externally driven. Because of the nature of decoherence, it is reasonable to expect that if further transforms besides $\mathcal{W}(n)$ are driven, the decoherence rate can only increase from what is derived below.

Suppose that there exists a field / qubit coupling of the form $\kappa E(t) \mathcal{S}_{x}$, where $E(t)$ is the electric field amplitude, and $\kappa$ is the field / qubit coupling. We assume the field uses only a single polarization for simplicity. If the qubits are magnetic dipoles, the form of the coupling is unchanged, with $B(t)$ substituted for $E(t)$. To give our programmer the greatest possible control, it is usually assumed that each qubit can be separately driven by the field. There are two ways to achieve this: spatial resolution (as is usually the case for lasers acting upon ions in traps) or frequency resolution (as is used in magnetic resonance). In either case, the Fourier components of the pulses acting upon separate qubits do not overlap. If spatial resolution is employed, then to prevent the pulses from overlapping, different directions of the beams are used. If frequency resolution is employed, then the pulses are centered at different frequencies in order to select a given transition. 
The entire qubit / field system is then described by a Hamiltonian of the form

$$
H / \hbar=\sum_{n} \omega_{n} \mathcal{S}_{z}(n)+\sum_{n, m} J_{n, m} \mathcal{S}_{z}(n) \mathcal{S}_{z}(m)+\ldots+\kappa \sum_{n} E_{n}\left(\vec{r}_{n}, t\right) \mathcal{S}_{x}(n)
$$

where the $J$ terms, and higher-order spin couplings if necessary, are present to implement the $\mathcal{R}$ and $\mathcal{O}$ transforms. The $\mathcal{W}(n)$ transform acting on qubit $n$ at position $\vec{r}_{n}$ is driven by the field $E_{n}$. Assume that the pulses have a square envelope with a center frequency $\bar{\omega}_{n}$, and that $\kappa E_{n}\left(\vec{r}_{n}, t\right) \gg J_{n, m}$ so that during a pulse we can ignore the $J$ couplings (but see note [20]). Then implementing $\mathcal{W}$ for each qubit amounts to finding the propagator for a single field / qubit interaction acting for a short time,

$$
H_{n} / \hbar=\omega_{n} \mathcal{S}_{z}(n)+\kappa E_{n}\left(\vec{r}_{n}\right) \cos \left(\bar{\omega}_{n} t\right) \mathcal{S}_{x}(n)
$$

We want to adjust these parameters so that we arrive at the result $\mathcal{W}(n)=\sqrt{2}\left(\mathcal{S}_{x}(n)-\mathcal{S}_{z}(n)\right)$. Suppose we write $\mathcal{W}(n)=\exp \left(-i \bar{\omega}_{n} \mathcal{S}_{z}(n) t\right) U_{n}(t)$, which amounts to transforming to a rotating frame with the field. Remove the rapidly oscillating terms to arrive at

$$
\frac{\partial}{\partial t} U_{n}=-i\left(\left(\omega_{n}-\bar{\omega}_{n}\right) \mathcal{S}_{z}(n)+\frac{\kappa}{2} E_{n}\left(\vec{r}_{n}\right) \mathcal{S}_{x}(n)\right) U_{n}
$$

The propagator for a time-independent Hamiltonian of the form $\vec{a} \cdot \overrightarrow{\mathcal{S}}$ is given by $\exp (-i \vec{a} \cdot \overrightarrow{\mathcal{S}} t)=$ $\cos (a t / 2)-2 i \sin (a t / 2) \vec{a} \cdot \overrightarrow{\mathcal{S}} / a$. In our case, $\vec{a}=\left\{\kappa E_{n}\left(\vec{r}_{n}\right) / 2,0, \omega_{n}-\bar{\omega}_{n}\right\}$, so to arrive at the correct result we require the field to be detuned below the qubit, $\bar{\omega}_{n}=\omega_{n}-\Omega / \sqrt{2}$, with a field strength $\kappa E_{n}\left(\vec{r}_{n}\right) / 2=-\Omega / \sqrt{2}$ and a pulse duration of $\pi / \Omega$. Then

$$
\mathcal{W}(n)=i \sqrt{2} \exp \left(-i \pi \frac{\bar{\omega}_{n}}{\Omega} \mathcal{S}_{z}(n)\right)\left(\mathcal{S}_{x}(n)-\mathcal{S}_{z}(n)\right)
$$


This is the form we seek, except for an extra phase factor. Since $\mathcal{W}(n)$ is applied uniformly to each qubit in the system, if the spread of frequencies of the separate qubits is not too large, it will be a constant factor for the entire quantum computer, and can be ignored. Otherwise, suitable delays built into the system can be implemented to achieve the same purpose.

\section{Inclusion of Quantum Back Reaction}

\subsection{Description of the Initial Field State.}

Before $t=0$, when the computation starts, the programmer creates pulses of the field that propagate towards and drive the qubits at separate times $t_{i}$. This is accomplished in a classical manner by turning on and off a classical current source, $j(\vec{r}, t)$, which interacts with the field through the vector potential, $H_{P}(t)=-\int d \vec{r} \mathcal{A}(\vec{r}, t) j(\vec{r}, t)$. For simplicity, a single polarization for $j$ is assumed. The current is classical in the sense that no quantum back reaction on the current source was included in the interaction Hamiltonian.

Grover's algorithm requires a series of pulses, created at different times, call them $t_{i}$, to drive each qubit $n$. Let the current distribution that drives qubit $n$ be $j_{n}(\vec{r}, t)$. It has significant Fourier

components over a range of $\vec{k} \in \mathrm{K}(n)$; as discussed previously, the $\mathrm{K}(n)$ are mutually disjoint sets. To create pulse $i$ in the series, turn $j_{n}$ on for a short time $\Delta t$ at time $t_{i}$. The field state is then transformed by $\exp \left(-i H_{P}\left(j_{n}\left(\vec{r}, t_{i}\right)\right) \Delta t / \hbar\right)=\prod_{\vec{k} \in \mathrm{K}(n)} D_{\vec{k}}\left(z_{\vec{k}}\right)$. The $D_{\vec{k}}(z)=\exp \left(z a_{\vec{k}}^{\dagger}+z^{\star} a_{\vec{k}}\right)$ are 
called displacement operators. They separately transform each mode of the field according to the complex-valued argument $z_{\vec{k}} \propto \Delta t \int j(\vec{r}, t) \exp (i \vec{k} \cdot \vec{r}) d \vec{r}$. The new version of Grover's algorithm then has the form $(\mathcal{W} \mathcal{R} \mathcal{W O})^{N} \sum_{x=0}^{2^{K}-1}|x\rangle \prod_{n}^{\text {qubits }} \prod_{t_{i}}^{\text {pulses }} \prod_{\vec{k} \in \mathrm{K}(n)} D_{\vec{k}}\left(z_{\vec{k}}\left(n, t_{i}\right)\right) \mid$ vac $\rangle$, where $\mid$ vac $\rangle$ indicates the vacuum state of the field. The $\mathcal{W}$ operators are now joint operators over the qubit and field.

The displacement operators produce coherent field states that have many classical properties. A list of some of these is given in Ref. [21]. We require the following properties: $D_{\vec{k}}^{\dagger}(z) D_{\vec{k}}(z)=$ $\mathbf{1}_{\vec{k}}$, and $D_{\vec{k}}^{\dagger}(z) a_{\vec{k}} D_{\vec{k}}(z)=a_{\vec{k}}+z$, and $D_{\vec{k}}^{\dagger}(z) a_{\vec{k}}^{\dagger} D_{\vec{k}}(z)=a_{\vec{k}}^{\dagger}+z^{\star}$. The above properties lead to the following useful identity. Define the positive and negative frequency electric field operators

$$
\mathcal{E}^{+}(\vec{r}, t)=\left(\mathcal{E}^{-}(\vec{r}, t)\right)^{\dagger}=\sum_{\vec{k}} i \sqrt{\frac{\hbar \omega}{2 \epsilon_{0} L^{3}}} a_{\vec{k}} e^{i(\vec{k} \cdot \vec{r}-\omega t)}
$$

The total electric field operator, $\mathcal{E}=\mathcal{E}^{+}+\mathcal{E}^{-}$, obeys Maxwell's operator equations for a source-free region. Given a function $f$ that can be represented by a Taylor expansion, then the following holds:

$$
\begin{gathered}
f\left(\mathcal{E}^{+}(\vec{r}, t), \mathcal{E}^{-}(\vec{r}, t)\right) \exp \left(-i H_{P}\left(j\left(\vec{r}^{\prime}, t_{i}\right)\right) \Delta t / \hbar\right) \\
=\exp \left(-i H_{P}\left(j\left(\vec{r}^{\prime}, t_{i}\right)\right) \Delta t / \hbar\right) f\left(\mathcal{E}^{+}(\vec{r}, t)+E^{+}(\vec{r}, t), \mathcal{E}^{-}(\vec{r}, t)+E^{-}(\vec{r}, t)\right)
\end{gathered}
$$

Although $f$ is still an operator, it contains the $c$-function $E^{+}(\vec{r}, t)$, which is the electric field profile that would be expected classically at position $\vec{r}$ and time $t$, resulting from a current distribution $j\left(\vec{r}^{\prime}, t_{i}\right)$ acting for a time $\Delta t$. 


\subsection{The Walsh-Hadamard Transform with a Quantum Field}

The goal of this section is to find a solution for a single qubit / field interaction, $\mathcal{W}(n)$. As before, we assume that the pulses have square envelopes with center frequency $\bar{\omega}_{n}$, and again let the detuning be given by $\Delta \omega=\omega_{n}-\bar{\omega}_{n}$. We also wish to distribute the displacement operators into Grover's algorithm in the following manner:

$$
\begin{gathered}
\prod_{n}\left\{\mathcal{W}(n)\left(t_{i}\right) \prod_{\vec{k} \in \mathrm{K}(n)} D_{\vec{k}}\left(z_{\vec{k}}\left(n, t_{i}\right)\right)\right\} \mathcal{R} \prod_{n}\left\{\mathcal{W}(n)\left(t_{i-1}\right) \prod_{\vec{k} \in \mathrm{K}(n)} D_{\vec{k}}\left(z_{\vec{k}}\left(n, t_{i-1}\right)\right)\right\} \mathcal{O} \cdots \\
\prod_{n}\left\{\mathcal{W}(n)\left(t_{2}\right) \prod_{\vec{k} \in \mathrm{K}(n)} D_{\vec{k}}\left(z_{\vec{k}}\left(n, t_{2}\right)\right)\right\} \mathcal{R} \prod_{n}\left\{\mathcal{W}(n)\left(t_{1}\right) \prod_{\vec{k} \in \mathrm{K}(n)} D_{\vec{k}}\left(z_{\vec{k}}\left(n, t_{1}\right)\right)\right\} \mathcal{O} \sum_{x=0}^{2^{K}-1}|x\rangle|\mathrm{vac}\rangle
\end{gathered}
$$

We have indicated the times at which the $\mathcal{W}(n)\left(t_{i}\right)$ interaction begins, e.g. the time at which the pulse arrives at the qubit. In commuting each $D$ past $\mathcal{R}, \mathcal{O}$, and $\mathcal{W}(m)$ with $m \neq n$, the $z_{\vec{k}}$ pick up a phase of $\exp (-i \omega t)$. This corresponds to the free propagation of the pulse while the other calculations occur. $D$ acting on qubit $n$ at a later time will not commute past $\mathcal{W}(n)$ at an earlier time. Classically, however, the pulse has not yet reached the qubit, so the expectation value of the $\mathcal{E}_{n}^{ \pm}$are zero. The non-zero commuter reflects the interaction of the qubit with the vacuum, including, e.g. spontaneous emission. Quantum systems are usually chosen explicitly to minimize this decoherence mechanism, so we assume that we can ignore it here.

Start with the interaction Hamiltonian of Eq. (3), except with the quantum field operators replacing the classical field. Transform to frames rotating with the field and qubit by assuming a solution of the form $\mathcal{W}(n)=\exp \left(-i H_{0}(n) t / \hbar\right) W$ where $H_{0}(n) / \hbar=\sum_{k \in \mathrm{K}(n)} \omega a_{\vec{k}}^{\dagger} a_{\vec{k}}+\omega_{n} \mathcal{S}_{z}(n)$ 
This results in an equation for $W$,

$$
\frac{d}{d t} W(t)=-i \frac{\kappa}{2}\left(\mathcal{S}_{+}(n) e^{i \omega_{n} t} \mathcal{E}_{n}^{+}\left(\vec{r}_{n}, t\right)+\mathcal{S}_{-}(n) e^{-i \omega_{n} t} \mathcal{E}_{n}^{-}\left(\vec{r}_{n}, t\right)\right) W(t)
$$

The modes over $\mathrm{K}(n)$ in the operator $\mathcal{E}_{n}^{ \pm}$slowly dephase during the interaction of the pulse with the qubit. This gives the effect of the pulse envelope on the qubit, but makes a solution somewhat difficult. However, our interest is to find only the lowest order departures from classical behavior. Here is one way to do this: first, insert a unit factor into the propagator, $\exp \left(-i H_{0}(n) t / \hbar\right) \prod_{\kappa(n)} D_{\vec{k}} D_{\vec{k}}^{\dagger}$ $W(t) \prod_{\mathrm{K}(n)} D_{\vec{k}}|\mathrm{vac}\rangle$. The $D$ to the right commutes to the front of the entire algorithm, and we solve for $U \equiv D^{\dagger} W D$ instead. From Eq. (7), this results in replacing $\mathcal{E}_{n}^{ \pm}$with $\mathcal{E}_{n}^{ \pm}+E_{n}^{ \pm}$in Eq. (8), where $E_{n}^{ \pm}$is the classical field at the qubit.

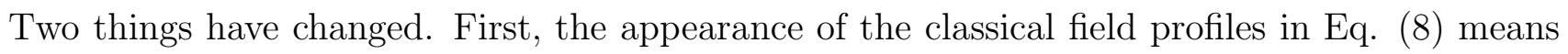
that part of $U$ alters the qubit evolution according to the classical field profile. Photons are still absorbed and emitted by the qubit, but the process occurs in such a way so that the coherent state of the field is not altered (which is a very "classical" behavior). Second, $U$ still contains field operators, but now they act upon the vacuum state of the field with the $D$ to the left. $U$ will create one-photon states (and higher orders as well) which are orthogonal to the vacuum state. Because $D$ is a unitary transform, the displaced one-photon states are orthogonal to the displaced vacuum states that describe the pulses. Thus, $U$ can entangle the qubit state with a new field state orthogonal to the original field state. This is precisely the description of a decoherence mechanism.

The idea that the classical field represents the lowest-order behavior suggests we should expand 
out $U$ as a series, $\sum_{j} U_{j}$, for which $j$ corresponds to the power of the quantum field operators (or equivalently, the power of $\sqrt{\hbar})$. This results in

$$
\frac{d}{d t} U_{0}=-i \frac{\kappa}{2}\left(E_{n}^{+} \mathcal{S}_{+}(n) e^{i \omega_{n} t}+E_{n}^{-} \mathcal{S}_{-}(n) e^{-i \omega_{n} t}\right) U_{0}
$$

for the classical behavior, and for $j \geq 1$,

$$
\frac{d}{d t} U_{j}=-i \frac{\kappa}{2}\left(E_{n}^{+} \mathcal{S}_{+}(n) e^{i \omega_{n} t}+E_{n}^{-} \mathcal{S}_{-}(n) e^{-i \omega_{n} t}\right) U_{j}-i \frac{\kappa}{2}\left(\mathcal{E}_{n}^{+} \mathcal{S}_{+}(n) e^{i \omega_{n} t}+\mathcal{E}_{n}^{-} \mathcal{S}_{-}(n) e^{-i \omega_{n} t}\right) U_{j-1}
$$

$U_{1}$ incorporates the field operators once, so it represents the lowest order quantum effects. Each $U_{j}$ has a solution of the form $\mathcal{S}_{\alpha} U_{\alpha, j}(t)+\mathcal{S}_{\beta} U_{\beta, j}(t)+\mathcal{S}_{+} U_{+, j}(t)+\mathcal{S}_{-} U_{-, j}(t)$,

$$
\begin{array}{ll}
\frac{d}{d t} U_{\alpha, 0}=-i \frac{\kappa}{2} e^{i \omega_{n} t} E_{n}^{+} U_{-, 0} & \frac{d}{d t} U_{\beta, 0}=-i \frac{\kappa}{2} e^{-i \omega_{n} t} E_{n}^{-} U_{+, 0} \\
\frac{d}{d t} U_{+, 0}=-i \frac{\kappa}{2} e^{i \omega_{n} t} E_{n}^{+} U_{\beta, 0} & \frac{d}{d t} U_{-, 0}=-i \frac{\kappa}{2} e^{-i \omega_{n} t} E_{n}^{-} U_{\alpha, 0}
\end{array}
$$

and for $j \geq 1$,

$$
\begin{aligned}
& \frac{d}{d t} U_{\alpha, j}=-i \frac{\kappa}{2} e^{i \omega_{n} t}\left(E_{n}^{+} U_{-, j}+\mathcal{E}_{n}^{+} U_{-, j-1}\right) \quad \frac{d}{d t} U_{\beta, j}=-i \frac{\kappa}{2} e^{-i \omega_{n} t}\left(E_{n}^{-} U_{+, j}+\mathcal{E}_{n}^{-} U_{+, j-1}\right) \\
& \frac{d}{d t} U_{+, j}=-i \frac{\kappa}{2} e^{i \omega_{n} t}\left(E_{n}^{+} U_{\beta, j}+\mathcal{E}_{n}^{+} U_{\beta, j-1}\right) \quad \frac{d}{d t} U_{-, j}=-i \frac{\kappa}{2} e^{-i \omega_{n} t}\left(E_{n}^{-} U_{\alpha, j}+\mathcal{E}_{n}^{-} U_{\alpha, j-1}\right)
\end{aligned}
$$

At this point we add in the classical field, whose envelope is a square pulse. Thus, let $E_{n}^{ \pm}=$ $E e^{\mp i \phi} e^{\mp i \bar{\omega} t}$, where $\phi$ is the phase, and $2 E$ is the field intensity. Let $\mathcal{L}_{ \pm}=(d / d t)^{2} \pm i \Delta \omega(d / d t)+$ $(\kappa E / 2)^{2}$ be linear differential operators. We can then rearrange Eq. (9) and (10) to read

$$
\mathcal{L}_{-} U_{\alpha, 0}=0, \quad \mathcal{L}_{+} U_{\beta, 0}=0, \quad \mathcal{L}_{-} U_{+, 0}=0, \quad \mathcal{L}_{+} U_{-, 0}=0
$$


and for $j \geq 1$,

$$
\begin{aligned}
\mathcal{L}_{-} U_{\alpha, j} & =-i \frac{\kappa}{2}\left(\frac{d}{d t}-i \Delta \omega\right)\left(e^{i \omega_{n} t} \mathcal{E}_{n}^{+} U_{-, j-1}\right)-\left(\frac{\kappa}{2}\right)^{2} E e^{-i \phi} e^{-i \bar{\omega}_{n} t} \mathcal{E}_{n}^{-} U_{\alpha, j-1} \\
\mathcal{L}_{+} U_{\beta, j} & =-i \frac{\kappa}{2}\left(\frac{d}{d t}+i \Delta \omega\right)\left(e^{-i \omega_{n} t} \mathcal{E}_{n}^{-} U_{+, j-1}\right)-\left(\frac{\kappa}{2}\right)^{2} E e^{i \phi} e^{i \bar{\omega}_{n} t} \mathcal{E}^{+} U_{\beta, j-1} \\
\mathcal{L}_{-} U_{+, j} & =-i \frac{\kappa}{2}\left(\frac{d}{d t}-i \Delta \omega\right)\left(e^{i \omega_{n} t} \mathcal{E}_{n}^{+} U_{\beta, j-1}\right)-\left(\frac{\kappa}{2}\right)^{2} E e^{-i \phi} e^{-i \bar{\omega}_{n} t} \mathcal{E}_{n}^{-} U_{+, j-1} \\
\mathcal{L}_{+} U_{-, j} & =-i \frac{\kappa}{2}\left(\frac{d}{d t}+i \Delta \omega\right)\left(e^{-i \omega_{n} t} \mathcal{E}_{n}^{-} U_{\alpha, j-1}\right)-\left(\frac{\kappa}{2}\right)^{2} E e^{i \phi} e^{i \bar{\omega}_{n} t} \mathcal{E}_{n}^{+} U_{-, j-1}
\end{aligned}
$$

The initial conditions are $U_{\alpha, 0}=U_{\beta, 0}=1$, and $U_{ \pm, 0}=0$, and $U_{j}=0$ for all $j \geq 1$; and for the first derivatives, $d U_{\alpha, 0} / d t=d U_{\beta, 0} / d t=0$, and $d U_{ \pm, 0} / d t=-i(\kappa / 2) E e^{\mp i \phi}$, and $d U_{\alpha, 1} / d t=d U_{\beta, 1} / d t=0$, and $d U_{ \pm, 1} / d t=-i(\kappa / 2) \mathcal{E}_{n}^{ \pm}\left(\vec{r}_{n}, 0\right)$, and $d U_{j} / d t=0$ for all $j \geq 2$. The solutions for $j=0$ are

$$
\begin{aligned}
& U_{\alpha, 0}=e^{i \Delta \omega t / 2}\left[\cos \theta t-i \frac{\sin \theta t}{\theta} \frac{\Delta \omega}{2}\right] \quad U_{\beta, 0}=e^{-i \Delta \omega t / 2}\left[\cos \theta t+i \frac{\sin \theta t}{\theta} \frac{\Delta \omega}{2}\right] \\
& U_{+, 0}=-i e^{i \Delta \omega t / 2}\left[\frac{\sin \theta t}{\theta}\right] \frac{\kappa}{2} E e^{-i \phi} \quad U_{-, 0}=-i e^{-i \Delta \omega t / 2}\left[\frac{\sin \theta t}{\theta}\right] \frac{\kappa}{2} E e^{i \phi}
\end{aligned}
$$

where the tip angle is given by $\theta=\sqrt{\Delta \omega^{2}+(\kappa E)^{2}} / 2$. This is a restatement of the usual expression for a Bloch vector influenced by a monochromatic field.

The solutions for $j=1$ are linear in the creation and annihilation operators. However, the field state they operate on is the vacuum state, so for the lowest order effect we will only require the solution for the creation operators. They are reasonably complex, so we simplify by setting $\Delta \omega$ $=\Omega / \sqrt{2}, \kappa E=-\Omega / \sqrt{2}$, and the pulse length to $\pi / \Omega$ to reproduce the classical Walsh-Hadamard 
transform. Then, to lowest order, and still in the doubly rotating frame, our transform is given by

$$
\frac{i}{\sqrt{2}}\left(\begin{array}{cc}
e^{-i \pi / \sqrt{8}} & e^{-i \pi / \sqrt{8}} e^{i \phi} \\
e^{i \pi / \sqrt{8}} e^{-i \phi} & -e^{i \pi / \sqrt{8}}
\end{array}\right)+\frac{\kappa}{4 \Omega} \sum_{\vec{k} \in \mathrm{K}(n)} \sqrt{\frac{\hbar \omega}{2 \epsilon_{0} L^{3}}}\left(\begin{array}{cc}
e^{-i \pi / \sqrt{8}} e^{-i \phi} g_{\beta} & e^{-i \pi / \sqrt{8}} g_{-} \\
e^{i \pi / \sqrt{8}} e^{2 i \phi} g_{+} & e^{i \pi / \sqrt{8}} e^{-i \phi} g_{\alpha}
\end{array}\right) e^{-i \vec{k} \cdot \vec{r}_{n}} a_{\vec{k}}^{\dagger}
$$

The functions that give the Fourier components for the shape of the one-photon "back-reaction field" are given by

$$
\begin{gathered}
g_{\alpha}=\frac{2 x^{2}+\frac{x}{\sqrt{2}}-1+\left(1+\frac{x}{\sqrt{2}}\right) e^{i \pi x}}{2 \sqrt{2} x\left(x^{2}-1\right)} \quad g_{\beta}=-\frac{1+\frac{x}{\sqrt{2}}+\left(2 x^{2}+\frac{x}{\sqrt{2}}-1\right) e^{i \pi x}}{2 \sqrt{2} x\left(x^{2}-1\right)} \\
g_{+}=-\frac{1+e^{i \pi x}}{4\left(x^{2}-1\right)} \quad g_{-}=\frac{(4 x+3 \sqrt{2})\left(1+e^{i \pi x}\right)}{4 \sqrt{2}\left(x^{2}-1\right)}
\end{gathered}
$$

for which $x \equiv\left(\omega-\bar{\omega}_{n}\right) / \Omega$, the offset of a field mode from the center frequency of the pulse, in units of the detuning.

The system is assumed to be contained in a resonator of size $L^{3}$. The resonator is not perfect, but has a finite bandwidth associated with it due to coupling and conductive losses, which allows for the passage of the classical square pulse (which has the Fourier components $\left.\left(e^{i \pi x}-1\right) / x\right)$. Because all the $g$ functions have significant Fourier components near $\bar{\omega}$ and decay as $x^{-1}$ like the square pulse, the resonator also allows the one-photon states created by the qubit to escape. The time-domain envelopes of these one-photon states (the Fourier transform of the $g$ functions) are shown in Fig. 11.

As in the classical field case, the extra phase factors of $\pm i \pi / \sqrt{8}$ in the transformation Eq. (14) 
can be dropped, since they can be compensated for by proper timing. Similarly, we assume $\phi=0$ at the start of the interaction. The $\phi$ term does indicate a curious feature of the back reaction: $g_{-}$ contains no term from the classical field, while $g_{+}$requires two interactions with the classical field in order to perturb the coherent state. This is consistent with the property that the removal of a photon from a coherent state does not alter it, while the addition of a photon does.

We can rewrite the transform Eq. (14) in terms of unitary field operators,

$$
\frac{i}{\sqrt{2}}\left(\begin{array}{cc}
1 & 1 \\
1 & -1
\end{array}\right)+\frac{\kappa}{4 \Omega} \sqrt{\frac{\hbar \bar{\omega}_{n}}{2 \epsilon_{0} L^{3}}}\left(\begin{array}{cc}
\sqrt{I_{\beta}} G_{\beta} & \sqrt{I_{-}} G_{-} \\
\sqrt{I_{+}} G_{+} & \sqrt{I_{\alpha}} G_{\alpha}
\end{array}\right)
$$

where the $G$ operators create normalized one-photon field states, so that $G^{\dagger} G=\mathbf{1}$. The normalization factors were found by numerical integration, for which the slowly varying $\sqrt{\omega}$ term was ignored: $I_{\alpha}=\int_{-\infty}^{\infty}\left|g_{\alpha}(x)\right|^{2} d x=4.297, I_{\beta}=4.297, I_{+}=0.617$, and $I_{-}=10.451$. Although the $G \mid$ vac $\rangle$ states are orthogonal to the initial coherent state, they are not mutually orthogonal. Later on, we will require their (non-normalized) overlap integrals $I_{\alpha, \beta}=\sqrt{I_{\alpha} I_{\beta}}\left\langle\operatorname{vac}\left|G_{\alpha}^{\dagger} G_{\beta}\right| \operatorname{vac}\right\rangle=\int_{-\infty}^{\infty} g_{\alpha}^{\star}(x) g_{\beta}(x) d x$ $=0.614+i 2.221, I_{\alpha,+}=-0.617+i 1.110, I_{\alpha,-}=4.300-i 3.331, I_{\beta,+}=0.617+i 1.110, I_{\beta,-}=$ $-4.300-i 3.331$, and $I_{+,-}=-1.850$.

The important result of this section is the transformation, Eq. (15). Each qubit / field interaction has a probability amplitude, proportional to $\Omega^{-1}$, to entangle the qubit with a field state orthogonal to the original field state. The greater $\Omega$, the lesser the decoherence via this mechanism. Actually, this may seem counterintuitive. Consider the interference pattern produced by a 
coherent beam of electrons incident upon a double slit. Now allow a laser to interact with one of the two paths the electron could travel from the slit to the detector. If photons are scattered out of the coherent modes into vacuum states, then the visibility of the interference pattern is degraded, as expected [10]. If, however, only stimulated processes are important, then the visibility of the interference pattern should increase as the laser intensity is increased. The Poisson statistics of a coherent state can more efficiently hide the information about which path the electron takes as the

number of photons in the beam increases. A similar situation has been noted with regard to welcher Weg experiments in atomic interferometry (see e.g. 22]).

\subsection{Grover's Algorithm with a Quantum Walsh-Hadamard Transform}

Taking the result from the previous section, Grover's algorithm is now

$$
\prod_{j}^{\text {steps }}\left[\prod_{n=1}^{K}\left(\mathbf{1}-\frac{i \kappa}{8 \Omega} \sqrt{\frac{\hbar \bar{\omega}_{n}}{\epsilon_{0} L^{3}}} \mathcal{A}\left(n, t_{j+1}\right)\right) \mathcal{W} \mathcal{R} \mathcal{W} \prod_{n=1}^{K}\left(\mathbf{1}-\frac{i \kappa}{8 \Omega} \sqrt{\frac{\hbar \bar{\omega}_{n}}{\epsilon_{0} L^{3}}} \mathcal{B}\left(n, t_{j}\right)\right) \mathcal{O}\right]\left(\frac{1}{\sqrt{2^{K}}} \sum_{x}|x\rangle\right)|\mathrm{vac}\rangle
$$

The displacement operators are not shown. They have been commuted all the way left, picking up the appropriate phase factors representing the propagation of the free field. The $\mathcal{A}$ and $\mathcal{B}$ are from transform Eq. (15), from which a factor of $\mathcal{W}(n)$ is first removed:

$$
\mathcal{A}=\left(\begin{array}{cc}
\sqrt{I_{\beta}} G_{\beta}+\sqrt{I_{-}} G_{-} & \sqrt{I_{\beta}} G_{\beta}-\sqrt{I_{-}} G_{-} \\
\sqrt{I_{+}} G_{+}+\sqrt{I_{\alpha}} G_{\alpha} & \sqrt{I_{+}} G_{+}-\sqrt{I_{\alpha}} G_{\alpha}
\end{array}\right) \quad \mathcal{B}=\left(\begin{array}{cc}
\sqrt{I_{\beta}} G_{\beta}+\sqrt{I_{+}} G_{+} & \sqrt{I_{-}} G_{-}+\sqrt{I_{\alpha}} G_{\alpha} \\
\sqrt{I_{\beta}} G_{\beta}-\sqrt{I_{+}} G_{+} & \sqrt{I_{-}} G_{-}-\sqrt{I_{\alpha}} G_{\alpha}
\end{array}\right)
$$


The fact that operators acting independently upon separate qubits and field modes mutually commute was also used.

When Eq. (16) is expanded out and only the lowest order terms are kept, to the original Grover's transform are added new terms. As previously discussed, the field operators in front of each term, one for each qubit and each pulse, create field states that are both mutually orthogonal, and orthogonal to the original field state. Thus, the probability that the final qubit state is $|y\rangle$ is the sum of the probabilities for each of separate terms to be in $|y\rangle$. In order to preserve normalization to $\mathcal{O}\left(\Omega^{-2}\right)$, the total probability to be in an orthogonal field state is subtracted from the probability of finding the field still in the original state at the end of the algorithm. The goal of this section is to calculate the success of this implementation of Grover's algorithm.

After $j$ successful steps of the algorithm, the computer state is given by

$$
\left(\frac{\cos (j \varphi)}{\sqrt{2^{K}-1}} \sum_{x \neq y}|x\rangle+\sin (j \varphi)|y\rangle\right)|\mathrm{vac}\rangle
$$

where $\sin \varphi=2 \sqrt{2^{K}-1} / 2^{K}$ [18]. The general trend for the influence of the back reaction can be discerned from the specific example of 3 qubits and $y=2$. A back reaction on the field can occur during either application of $\mathcal{W}$. If it occurs immediately after $\mathcal{O}$ has been called (the operator $\mathcal{B}$ ), or after the invert about average transform (the operator $\mathcal{A}$ ). Suppose the reaction occurs after $\mathcal{O}$, and it is for the least significant qubit. Then the computer state becomes

$$
\left\{\frac { \operatorname { c o s } ( j \varphi ) } { \sqrt { 2 ^ { K } - 1 } } \left(\left(B_{\beta}|0\rangle+B_{+}|1\rangle\right)+\left(B_{\alpha}|1\rangle+B_{-}|0\rangle\right)+\left(B_{\alpha}|3\rangle+B_{-}|2\rangle\right)+\left(B_{\beta}|4\rangle+B_{+}|5\rangle\right)+\right.\right.
$$




$$
\left.\left.\left(B_{\alpha}|5\rangle+B_{-}|4\rangle\right)+\left(B_{\beta}|6\rangle+B_{+}|7\rangle\right)+\left(B_{\alpha}|7\rangle+B_{-}|6\rangle\right)\right)-\sin (j \varphi)\left(B_{\beta}|2\rangle+B_{+}|3\rangle\right)\right\}|\operatorname{vac}\rangle
$$

The amplitudes of pairs of states that differ at their least significant digit such as $(0,1),(2,3)$, and so on, are mixed. If the error occurs for the second least significant qubit, then

$$
\begin{gathered}
\left\{\frac { \operatorname { c o s } ( j \varphi ) } { \sqrt { 2 ^ { K } - 1 } } \left(\left(B_{\beta}|0\rangle+B_{+}|2\rangle\right)+\left(B_{\alpha}|1\rangle+B_{-}|3\rangle\right)+\left(B_{\alpha}|3\rangle+B_{-}|1\rangle\right)+\left(B_{\beta}|4\rangle+B_{+}|6\rangle\right)+\right.\right. \\
\left.\left.\left(B_{\alpha}|5\rangle+B_{-}|7\rangle\right)+\left(B_{\beta}|6\rangle+B_{+}|4\rangle\right)+\left(B_{\alpha}|7\rangle+B_{-}|5\rangle\right)\right)-\sin (j \varphi)\left(B_{\beta}|2\rangle+B_{+}|0\rangle\right)\right\}|\operatorname{vac}\rangle
\end{gathered}
$$

The difference is in which pairs of states are mixed, and whether the qubit involved in the back reaction was initially in state 0 or 1 . Using this, we can sum over all the qubits of the computer, the total probability for a back reaction to occur during the first of the two applications of $\mathcal{W}$, at step $j$ in the algorithm. Suppose that the qubit frequencies are sufficiently close so that all pulse center frequencies are $\bar{\omega}$. Then

$$
\begin{gathered}
\left.\left.\left(\frac{\kappa}{8 \Omega}\right)^{2} \frac{\hbar \bar{\omega}}{\epsilon_{0}}\left[\left.\frac{K}{2} \frac{2^{K}-2}{2^{K}-1} \cos ^{2}(j \varphi)\left(\left|\left(B_{\beta}+B_{-}\right)\right| \mathrm{vac}\right\rangle\right|^{2}+\left|\left(B_{\alpha}+B_{+}\right)\right| \mathrm{vac}\right\rangle\right|^{2}\right)+ \\
\left.\left.(K-\|y\|)\left(\left|\frac{\cos (j \varphi)}{\sqrt{2^{K}-1}} B_{-}\right| \mathrm{vac}\right\rangle-\left.\sin (j \varphi) B_{\beta}|\mathrm{vac}\rangle\right|^{2}+\left|\frac{\cos (j \varphi)}{\sqrt{2^{K}-1}} B_{\alpha}\right| \mathrm{vac}\right\rangle-\left.\sin (j \varphi) B_{+}|\mathrm{vac}\rangle\right|^{2}\right)+ \\
\left.\left.\left.\|y\|\left(\left|\frac{\cos (j \varphi)}{\sqrt{2^{K}-1}} B_{\beta}\right| \mathrm{vac}\right\rangle-\left.\sin (j \varphi) B_{-}|\mathrm{vac}\rangle\right|^{2}+\left|\frac{\cos (j \psi)}{\sqrt{2^{K}-1}} B_{+}\right| \mathrm{vac}\right\rangle-\left.\sin (j \varphi) B_{\alpha}|\mathrm{vac}\rangle\right|^{2}\right)\right]
\end{gathered}
$$

The factor $\|y\|$ appears since the back reaction depends upon whether a qubit was initially in state 0 or 1 . Now suppose $K$ is large enough so that $2^{K} \gg 1$. The following approximations are useful: $\sum_{j=1}^{\pi / 2 \varphi} \cos ^{2}(j \varphi) \approx \int_{0}^{\pi / 2} \cos ^{2} x d x / \varphi=\pi / 4 \varphi$, and $\sum_{j=1}^{\pi / 2 \varphi} \sin ^{2}(j \varphi) \approx \pi / 4 \varphi$, and $\sum_{j=1}^{\pi / 2 \varphi} \sin (j \varphi) \cos (j \varphi)$ 
$\approx 1 / 2 \varphi$. Again, keeping only the largest terms in $K$, the probability that a back reaction occurs for any qubit, and at any step, during the first of the two $\mathcal{W}$ transforms is given by

$$
\begin{gathered}
\left.\left.\left(\frac{\kappa}{8 \Omega}\right)^{2} \frac{\hbar \bar{\omega}}{\epsilon_{0}} \frac{\pi}{8} 2^{K / 2}\left[\left.\frac{K}{2}\left(\left|\left(B_{\beta}+B_{+}\right)\right| \mathrm{vac}\right\rangle\right|^{2}+\left|\left(B_{\alpha}+B_{+}\right)\right| \mathrm{vac}\right\rangle\right|^{2}\right) \\
\left.\left.\left.\left.\left.+\left.(K-\|y\|)\left(\left|B_{\beta}\right| \mathrm{vac}\right\rangle\right|^{2}+\left|B_{+}\right| \mathrm{vac}\right\rangle\left.\right|^{2}\right)+\left.\|y\|\left(\left|B_{-}\right| \mathrm{vac}\right\rangle\right|^{2}+\left|B_{\alpha}\right| \mathrm{vac}\right\rangle\left.\right|^{2}\right)\right]
\end{gathered}
$$

If the back reaction occurs during the second $\mathcal{W}$ in an iteration, then $j$ starts at 2 (the first invertabout-average step is always carried out), and the sign of the amplitude for $|y\rangle$ is positive. Taking the limit for large $K$, the cross-terms that depend upon the sign of $a_{y}$ drop out, and the final expression is the same as above except with the $B$ operators replaced by those of $A$. The total probability of a back reaction anywhere in the algorithm is then the sum of these two. Starting with the operators of Eq. (17), the matrix elements are expressed in terms of the normalization and overlap of the $G|\mathrm{vac}\rangle$ states, e.g. $\left.\left.\left|\left(B_{\beta}+B_{-}\right)\right| \mathrm{vac}\right\rangle\left.\right|^{2}+\left|\left(B_{\alpha}+B_{+}\right)\right| \mathrm{vac}\right\rangle\left.\right|^{2}=2\left(I_{\alpha}+I_{\beta}+I_{+}+I_{-}\right)$ $+4 \Re e\left(I_{\beta,-}+I_{\alpha,+}\right)$. Plugging in, the final probability to end up entangled with an orthogonal field state is

$$
\left(\frac{\kappa}{\Omega}\right)^{2} \frac{\hbar \bar{\omega}}{\epsilon_{0}} \sqrt{2^{K}}(0.211 K+0.241\|y\|) .
$$

How much do the orthogonal field states contribute to the correct final answer? First, note that the matrix elements of $\mathcal{A}$ and $\mathcal{B}$ all have similar magnitudes. Thus, they equally mix the state $|y\rangle$ with the state connected to it by flipping the qubit that experiences the back reaction. Early in the algorithm when $\sin (j \varphi) \ll 1$, this does not increase the amplitude in $a_{y}$ significantly, since the 
state that mixes with $|y\rangle$ has amplitude $\cos (j \varphi) / \sqrt{2^{K}} \ll 1$. At later times, however, the amplitude of $|y\rangle$ is near 1, so the back reaction decreases the probability to be in state $|y\rangle$ by roughly half.

Thus, after $j$ iterations of Grover's algorithm, a back reaction will cause the result to appear as if only $\approx j / 2$ iterations had been performed. Further iterations will continue to transform these entangled states, but this does not necessarily improve the final result. Recall from Eq. (1) that amplitude is rotated into $|y\rangle$ only when the signs of $a_{y}$ and $\sum_{x \neq y}|x\rangle$ are the same. For large $K$, the

amplitude $a_{y}$ is $\approx \sin (j \varphi)\left(\sqrt{I_{\beta}} G_{\beta}+\sqrt{I_{-}} G_{-}\right) \mid$vac $\rangle$. The other qubit states are entangled with field states that are partly orthogonal to this state, but the amplitude that lies along the same direction in the Hilbert space of the field is, for large $K, \cos (j \varphi)\left(I_{\beta}+I_{\beta,+}+I_{-, \beta}+I_{-,+}\right) / \sqrt{I_{\beta}+I_{-}+2 \Re e\left(I_{\beta,-}\right)}$ $=(-2.232+i 0.448) \cos (j \varphi)$. The real part has switched sign, and so further iterations will actually remove amplitude from $|y\rangle$. It should be clear that the back reaction essentially scrambles the memory of the quantum computer, from which further iterations will not, in general, recover the correct amplitude before the computation ends.

\section{Quantum Back Reaction for the CNOT Gate}

In this section, we briefly examine what happens when a classical field is used to drive a logic gate, such as $\mathcal{R}$, that entangles together separate qubits. As before, let us focus on a single example, that of the controlled-NOT (CNOT) gate. CNOT is defined as a transform acting on two qubits 
that flips the second qubit only if the first qubit is $1:|00\rangle \rightarrow|00\rangle,|01\rangle \rightarrow|01\rangle,|10\rangle \rightarrow|11\rangle$, and $|11\rangle \rightarrow|10\rangle$. As for the Walsh-Hadamard transform, there are numerous ways to implement the CNOT gate [5]. Perhaps the most straight forward method is the use of a selective pulse. What we mean by this is the following.

The two qubits to be transformed by CNOT have a Hamiltonian

$$
H / \hbar=\omega_{1} \mathcal{S}_{z}+\omega_{2} \mathcal{I}_{z}+J \overrightarrow{\mathcal{S}} \cdot \overrightarrow{\mathcal{I}}+\sum_{\vec{k}} \omega a_{\vec{k}}^{\dagger} a_{\vec{k}}+\kappa\left(\mathcal{S}_{x}+\mathcal{I}_{x}\right)\left(\mathcal{E}^{+}+\mathcal{E}^{-}\right)
$$

By $\overrightarrow{\mathcal{S}} \cdot \overrightarrow{\mathcal{I}}$ we mean the qubit operator $\mathcal{S}_{x} \mathcal{I}_{x}+\mathcal{S}_{y} \mathcal{I}_{y}+\mathcal{S}_{z} \mathcal{I}_{z}$. Assume that $\omega_{2}>\omega_{1} \gg\left|\omega_{1}-\omega_{2}\right| \gg$ $J$. In this case, the transitions driven by the field occur at $\approx \omega_{1} \pm J / 2+J^{2} / 4\left(\omega_{1}-\omega_{2}\right)$ and $\approx$ $\omega_{2} \pm J / 2-J^{2} / 4\left(\omega_{1}-\omega_{2}\right)$, They appear as a "doublet of doublets", that is, two sets of two lines each. Since $H$ is nearly diagonal for small $J$, the highest frequency transition corresponds to $|10\rangle \leftrightarrow|11\rangle$. Thus, a CNOT gate can be implemented by selectively inverting this transition with a square pulse of duration $T \gg 2 \pi / J$. ( $T$ also depends on the matrix element for the transition.) The long length of the pulse keeps its bandwidth small enough so that it does not significantly overlap with the other transitions in the system.

As before, first assume that the pulse is described by a coherent field state, and commute the displacement operator to the left. This replaces $\mathcal{E}^{ \pm}$with $\mathcal{E}^{ \pm}+E e^{\mp i \bar{\omega} t}$ in Eq. (22). Second, split $H=H_{C}+H_{Q}$, where $H_{Q}$ contains the electric (or magnetic) field operators terms. Suppose we find $U_{C}(t)$, the propagator corresponding to $H_{C}$. Then the total propagator for the system can be 
formally integrated as

$U(t)=U_{C}(t)-\frac{i}{\hbar} \int_{0}^{t} d t^{\prime} U_{C}\left(t-t^{\prime}\right) H_{Q} U_{C}\left(t^{\prime}\right)-\frac{1}{\hbar^{2}} \int_{0}^{t} d t^{\prime} \int_{0}^{t^{\prime}} d t^{\prime \prime} U_{C}\left(t-t^{\prime}\right) H_{Q} U_{C}\left(t^{\prime}-t^{\prime \prime}\right) H_{Q} U_{C}\left(t^{\prime \prime}\right)+\cdots$

Computing the value of the first integral in this series is the goal of this section. As before, this integral can be physically interpreted as follows: the qubit evolves under $U_{C}$, coherently evolving under the influence of a classical field until time $t^{\prime}$, when a scattering event occurs due to the quantum nature of the field. After the scattering, the qubit / field system evolves as before until final time $t$. Higher order corrections then correspond to multiple scatterings (or, multiple field operators). To find $U_{C}$, first assume a solution of the form $\exp \left(-i \sum \omega a_{\vec{k}}^{\dagger} a_{\vec{k}}\right) \exp \left(-i \bar{\omega}\left[\mathcal{S}_{z}+\mathcal{I}_{z}\right] t\right)$ $U_{C 1}$, and then drop the rapidly oscillating terms. Then diagonalize the resulting Hamiltonian,

$$
H_{C 1}=\left(\begin{array}{cccc}
\left(\omega_{1}+\omega_{2}\right) / 2-\bar{\omega}+J / 4 & \kappa E / 2 & \kappa E / 2 & 0 \\
\kappa E / 2 & \left(\omega_{1}-\omega_{2}\right) / 2-J / 4 & J / 2 & \kappa E / 2 \\
\kappa E / 2 & J / 2 & \left(-\omega_{1}+\omega_{2}\right) / 2-J / 4 & \kappa E / 2 \\
0 & \kappa E / 2 & \kappa E / 2 & -\left(\omega_{1}+\omega_{2}\right) / 2+\bar{\omega}+J / 4
\end{array}\right),
$$

to find eigenvalues $\lambda_{n}$ and eigenvectors $v_{n}$. Plugging in the $U_{C}$ operator into Eq. (23), and as before keeping only the terms linear in the creation operators, the lowest order quantum back reaction is (in the rotating frame)

$$
-\frac{\kappa}{2} \sum_{\vec{k}} e^{i \vec{k} \cdot \vec{r}} \sqrt{\frac{\hbar \omega}{2 \epsilon_{0} L^{3}}} a_{\vec{k}}^{\dagger} \sum_{n, m=1}^{4} v_{n} v_{n}^{\dagger}\left(\mathcal{S}_{-}+\mathcal{I}_{-}\right) v_{m}^{\dagger} v_{m} e^{i \lambda_{n} T} \frac{e^{i\left(\omega-\bar{\omega}+\lambda_{m}-\lambda_{n}\right) t}-1}{i\left(\omega-\bar{\omega}+\lambda_{m}-\lambda_{n}\right)}
$$


The real parts of the field amplitudes of the one-photon states entangled with different qubit states for a few of the matrix elements of $U$ are shown in Fig. 2. The parameters used to generate the figure were $\omega_{1}=20, \omega_{2}=21$, and $J=0.4$ for the qubits, and $\kappa E=0.01$, and $T=100.6$ for the classical pulse profile. The classical result, $U_{C}(T)$, transfers $97 \%$ of the amplitude between states $|11\rangle$ and $|10\rangle$. These parameters are not meant to represent any specific spectroscopic method.

For on-resonant transitions, the matrix element peaks strongly at $T \propto E^{-1}$. Thus, as before, weaker pulses lead to greater decoherence. The matrix element for the transition $|01\rangle \rightarrow|00\rangle$ is found to have the greatest magnitude. It may be surprising that transitions that are not being driven by the external field can also have large decoherence rates. Recall that this method of implementing the CNOT gate actually drives all the allowed transitions, but is only resonant with one of them. This decoherence process continues to grow because these other transitions that can lead to photon emission are weakly but continuously excited.

In summary, several conclusions are of interest. First, the field states that become entangled with the two-qubit states are orthogonal to the initial field state, but they are not mutually orthogonal. In fact, their projections onto one another have essentially random phases. Computations that use the phases of the qubit state will be scrambled by this process. Secondly, because the CNOT gate involves entangling separate qubits, the scattering process also involves creating mutually entangled qubit / field states. It appears reasonable to suggest that this kind of field / multiple qubit entanglement will result when such logic gates are driven by external fields. 


\section{Discussion}

\subsection{Grover's algorithm}

When unitary transforms are driven by externally generated coherent fields in the manner discussed above, a decoherence mechanism exists that, with each applied pulse, tends to scramble the computer's memory. This decoherence mechanism is slightly different from the usual environmentally induced decoherence, in that it increases as the number of times the programmer attempts to manipulate the qubit system coherently. In the case of Grover's search algorithm where the Walsh-Hadamard transforms are externally driven, the degradation of the correct response scales as $0.2 K 2^{K / 2}\left(\hbar \bar{\omega} / \epsilon_{0} L^{3}\right)(\kappa / \Omega)^{2}$

A criticism of this analysis might be in the specific choice used to implement $\mathcal{W}(n)$. Whatever method is chosen, the field / qubit propagators still hold, and some back reaction must exist (but see below). In general, the degradation should scale as the number of times a qubit transform is driven. For Grover's algorithm, if no error correction routines are implemented, then the amplitude $\Omega$ of the field will have to increase exponentially with increasing $K$ in order to keep the error below a fixed bound. Clearly, this is not a scalable way to implement Grover's algorithm.

How important is this decoherence mechanism to the different proposed quantum computer schemes? Let us employ simple order of magnitude arguments, and ignore for the moment the implementation of error-correcting codes. The total probability for decoherence can also be written 
as $0.2\left[(\hbar \bar{\omega}) /\left(L^{3} \epsilon_{0} E^{2} / 2\right)\right] K 2^{K / 2}$. The pre-factor is the ratio of the energy of a single photon, to the total energy in the pulse (energy density times resonator volume), or the reciprocal of the total number of photons in the resonator used to create a pulse.

First, examine the case for using lasers to drive single ions or atoms. A recent experimental demonstration of a logic gate using trapped ${ }^{9} \mathrm{Be}^{+}$ions as qubits [23] used $1 \mathrm{~mW}$ pulses of $\approx 10^{-4}$ s duration at $300 \mathrm{~nm}$. This corresponds to $10^{12}$ photons per pulse. The very small pre-factor will not pose a problem for computations involving a polynomial number of steps with increasing $K$, but for Grover's algorithm this mechanism limits the number of qubits to $\approx 70$.

Next, suppose we examine the case for NMR quantum computing. First, let us address the question of utilizing the signal from a large number of independent quantum computers. Let us assume that our sample can be prepared in the ground state (all spins initially down), and let us ignore the interaction of the computers with one another through the field. We note that these assumptions are hardly justified for real world systems, but assuming a finite temperature for bulk quantum computing causes separate difficulties that have been addressed elsewhere 24. The application of Grover's algorithm results in a final state

$$
|\Psi\rangle=\prod_{i=1}^{10^{23}}\left(\left(1-\gamma^{2} / 2\right)\left|y^{(i)}\right\rangle+\gamma \sum_{x} G(x)\left|x^{(i)}\right\rangle\right)|\mathrm{vac}\rangle
$$

where $G$ creates orthogonal field states that contribute little amplitude to the correct solution. The total signal is the sum from all of the quantum computers in the sample, $\left\langle\Psi\left|\sum_{i}\left(\left|y^{(i)}\right\rangle\left\langle y^{(i)}\right|\right)\right| \Psi\right\rangle=\mathrm{N}$ $\left(1-\gamma^{2} / 2\right)$. The point is that at low temperatures, the macroscopic decoherence rate is multiplied by 
the total number of independent quantum computers in the sample. Typically, NMR uses $\nu \approx 10^{8}$ $\mathrm{Hz}$, or a photon energy of $10^{-25} \mathrm{~J}$. Pulses are $100 \mathrm{~W}$ for $10 \mu \mathrm{s}$, for a total energy of $10^{-3} \mathrm{~J}$. Thus, there are $10^{22}$ photons per pulse. This limits Grover's algorithm for NMR to $\approx 140$ qubits if we can do NMR on a single spin system; but for a micro-mole $\left(10^{17}\right)$ of quantum computers, we are limited to roughly 25 qubits. If electron spin resonance is substituted as the spectroscopic technique, then $\nu \approx 10^{10} \mathrm{~Hz}$, pulses are $1 \mathrm{~kW}$ for $10 \mathrm{~ns}$, and thus require $10^{18}$ photons, a negligible improvement over NMR.

If, as is usually the case, $\Omega \ll \omega_{n}$, then the number of photons required to generate $\mathcal{W}(n)$ is proportional to $\Omega /\left(\kappa^{2} \omega_{n}\right)$. Thus, physical systems with small values of $\omega_{n}$ or $\kappa$ are the most resistant to the above decoherence mechanism. Unfortunately, such systems have other limitations: if $\omega_{n}$ is small, then the temperature of the system is required to scale with increasing $K$ in an unfortunate manner [24], while $\kappa$ is small, then the time required to drive the qubits increases, which in turn slows the entire computation down. It seems that quantum computers driven or programmed by externally applied fields will face significant design trade-offs.

Will driving qubits by externally applied, static electric fields [ [ $[$ offer any significant advantages? (Similar proposals might be envisioned for NMR by applying different static magnetic fields along different directions to individual spins.) In the Coulomb gauge, such longitudinal fields are not independent degrees of freedom [25]. Instead, the total Hamiltonian of such a system must include the particles whose charges give rise to the static fields. Thus, for the case of qubits driven by 
electrostatic fields from electrodes, decoherence results from the field operators (now with half integer spin statistics) for the Fermi levels of each electrode becoming entangled with the qubit operators. Finding the decoherence rate in this case is complicated by the extra structure for the electrons in the electrode, but it would be surprising if some decoherence mechanism was not present.

\subsection{Error Correcting Codes}

Finally, we briefly examine some possible means to reduce the decoherence, and some difficulties they may encounter because of the unusual nature of this decoherence mechanism. First, consider the use of error-correcting codes. They are a general method by which to reduce decoherence [26, 27], provided that the decoherence is a statistically independent process amongst the qubits within each code-word. For logic gates applied bit-wise to code-words [26], these error correcting codes should be able to correct for this decoherence as for any other environmentally induced decoherence. The difficulty comes in when one considers how to actually implement the detection and correction scheme. Because of the requirement that errors within code-words be independent between qubits, we can not expect to have qubit / qubit couplings within each code-word. For the encoding schemes for which the authors are aware, this seems to require the "ancillary" bits [26] to be driven by external fields in such a way so that multiple qubits interact with the ancillary bit at once. Doing this with external fields, however, leads to entanglements between the qubits 
within the code-word and the external fields. This in turn means that errors developed during the error correction scheme itself will not have the required statistical independence. However, in order to better understand how this decoherence mechanism might influence error correcting schemes, it would be helpful to examine a complete program, such as Grover's algorithm with a specific code implemented.

Perhaps more hopefully, it appears that there are independent means by which storing information in multiple qubits could reduce this decoherence mechanism. Note that the appearance of terms such as $\left|B_{\beta}+B_{-}\right|$in Eq. (20) shows that destructive interference can lessen the probability of a back reaction. It is known to be possible to quench spontaneous emission in multi-level systems [28], which is a process quite similar to the decoherence mechanism considered here. Thus, it seems likely that a similar design could be used to remove the lowest order terms in the expansion of Eq. (23). Then, the decoherence rate increases as the inverse square of the number of photons in a pulse, which can be considered as a significant improvement.

\section{Conclusions}

Quantum computers that use external, classically generated electromagnetic fields to drive the evolution of the system undergo a decoherence induced by the quantum back reaction to those fields. The probability for the quantum system to be degraded increases as the total number of externally driven transforms, and inversely as the number of photons in the pulse. For algorithms in which no error-correcting codes are implemented, and for which the number of pulses is required to 
increase exponentially as the problem size increases, the field intensity driving the system will also be required to increase exponentially in order to bound the degradation of the response. And for the implementation of error-correcting codes, the use of external fields for error detection and correction gives rise to a decoherence that does not have the property of being statistically independent between separate qubits in the code-word.

\section{Acknowledgements}

We gratefully acknowledge support from the Air Force Office of Scientific Research. 


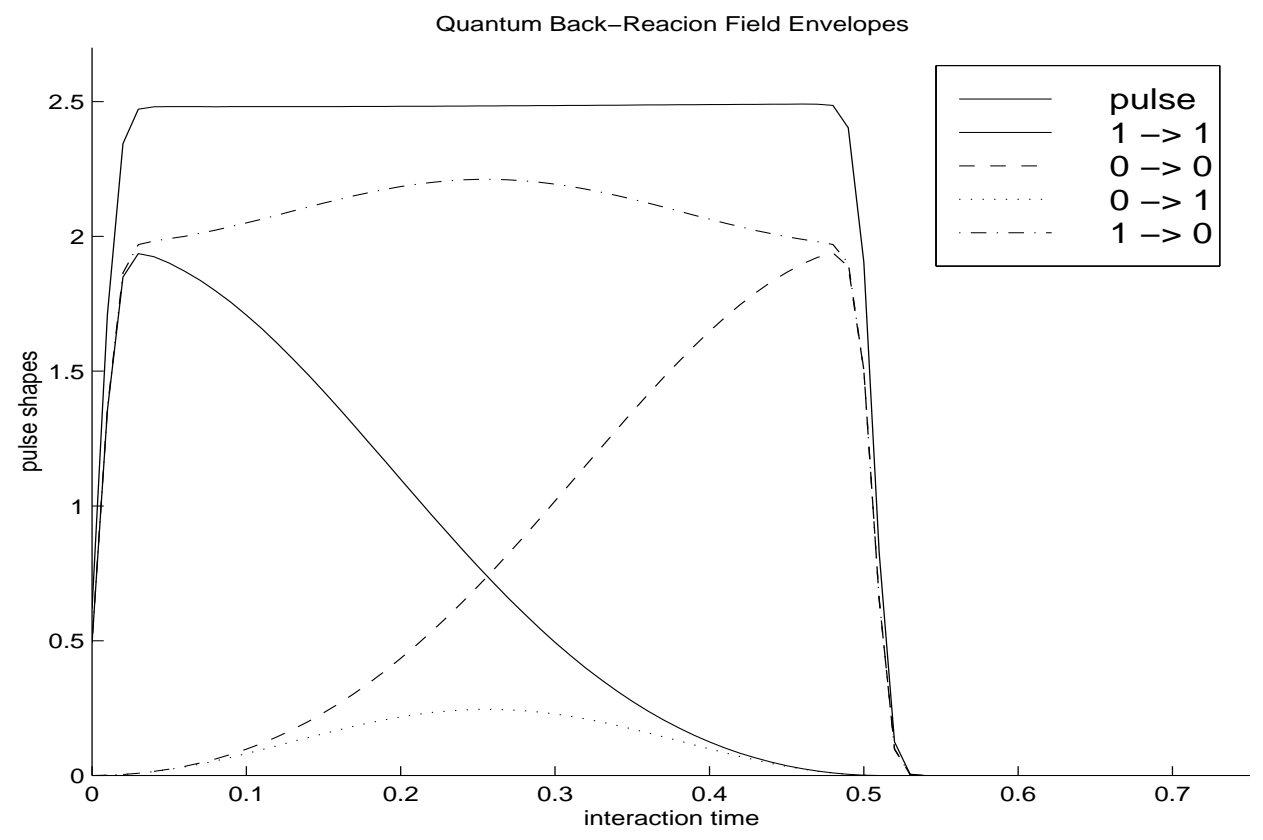

Figure 1: FIG. 1. A plot of the envelope of the field that describes the one-photon state emitted by a qubit whose state changes as indicated. For comparison, the envelope of the classical pulse is also shown. Time is in units of $\Omega$. 


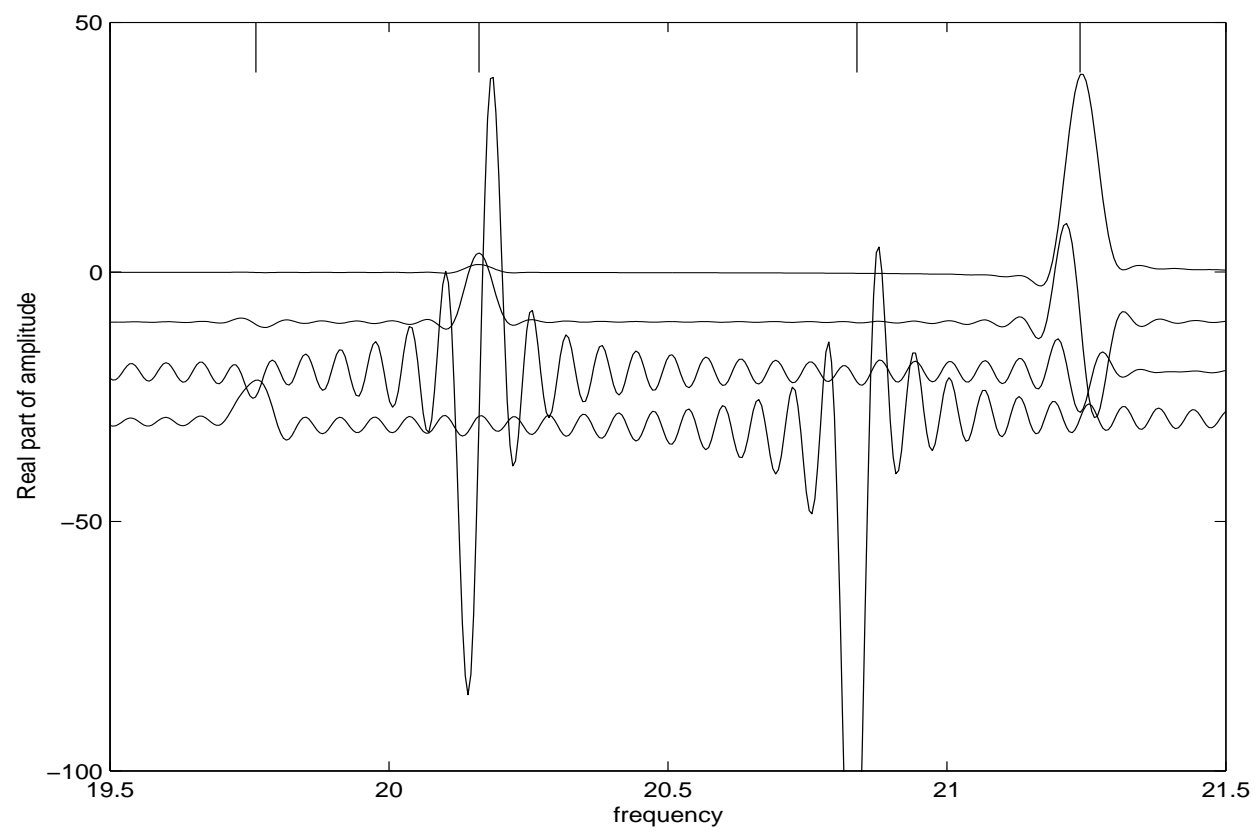

Figure 2: FIG 2. A plot of the real part of the frequency-dependent matrix elements for transitions (from top to bottom) $|11\rangle \rightarrow|11\rangle,|11\rangle \rightarrow|10\rangle,|10\rangle \rightarrow|01\rangle$, and $|01\rangle \rightarrow|00\rangle$. The lines at top represent the transitions in the two-qubit system driven by weak fields.

\section{References}

[1] R. Feynman, Found. Phys. 16, 507 (1986).

[2] D Deutsch, Proc. R. Soc. London A 425, 73 (1989).

[3] S Lloyd, Science 261, 1569 (1993). 
[4] J. A. Jones, M. Mosca, J. Chem. Phys. 109, 1648 (1998).

[5] D. G. Cory, A. F. Fahmy, and T. F. Havel, in Proceedings of the Fourth Workshop on Physics and Computation, edited by T. Toffoli (New England Complex Systems Institute, Boston, 1996), pp.87-91.

[6] J. I. Cirac, P. Zoller, Phys. Rev. Lett. 74, 4091 (1995).

[7] S. Schneider, D. F. V. James, and G. J. Milburn, e-print quant-ph/9808012.

[8] B. E. Kane, Nature 393, 133 (1998).

[9] D. S. Chemla, D. A. B. Miller, in Heterojunction Band Discontinuities, Physics, and Device Applications., edited by F. Capasso and G. Margaritondo (North-Holland, Elsevier, New York, 1987).

[10] R. Feynman, Leighton, Sands, The Feynman Lectures on Physics, Volume III (Addison-Wesley, Reading, MA, 1965). Ch. 3.

[11] D. Giulini et al., Decoherence and the Appearance of a Classical World in Quantum Theory (Springer, Berlin, 1996).

[12] W. Zurek, Phys. Today 44, 36 (1991).

[13] L. Grover, Phys. Rev. Lett. 79, 325 (1997). 
[14] N. Imoto, Prog. Crystal Growth and Charact. 33, 295 (1996).

[15] M. R. Garey and D. S. Johnson, Computers and Intractability. A Guide to the Theory of NP Completeness. (W. H. Freeman, New York, 1979).

[16] C. H. Bennet, E. Bernstein, G. Brassard, and U. Vazirani, e-print quant-ph/9701001.

[17] M. Boyer et al., e-print quant-ph/9605034.

[18] C. Zalka, e-print quant-ph/9711070.

[19] R. R. Ernst, G. Bodenhausen, and A. Wokaun, Principals of Nuclear Magnetic Resonance in One and Two Dimensions. (Clarendon Press, Oxford, 1987).

[20] The assumption $\kappa E \gg J$ is useful to implement single qubit interactions only. In NMR terminology, it corresponds to the transformation of magnetization from a given spin, irregardless of J-J couplings (or hyperfine couplings for the case of ESR). Implementing transforms that entangle separate qubits, however, can involve the use of selective pulses that manipulate the magnetization within the J-J coupling (or hyperfine couplings) manifold, as shown for the CNOT gate.

[21] L. Mandel and E. Wolf, Optical Coherence and Quantum Optics. (Cambridge University Press, Cambridge, 1995). Ch. 11.13.

[22] M. O. Scully, B.-G. Englert, and H. Walther, Nature 351, 111 (1991). 
[23] C. Monroe et al., Phys. Rev. Lett. 75, 4714 (1995).

[24] W. S. Warren, Science 277, 1688 (1997).

[25] C. Cohen-Tannoudji, J. Dupont-Roc, and G. Grynberg, Photons 83 Atoms: Introduction to Quantum Electrodynamics. (Wiley, New York, 1989).

[26] P. Shor, in Proceedings, 35th Annual Symposium on Foundations of Computer Science. (IEEE Press, New York, 1994), pp.56-65.

[27] E. Knill and R. Laflamme, Phys. Rev. A 55, 900 (1997).

[28] H. Lee, P. Polynkin, M. O. Scully, and S.-Y. Zhu, Phys. Rev. A 55, 4454 (1997). 